\title{
Chronic cough - assessment of treatment efficacy based on two questionnaires
}

\author{
Marta Dąbrowska ${ }^{1}$ Elżbieta M. Grabczak ${ }^{1}$, Magdalena Arcimowicz², Anna Domeracka-Kołodziej², \\ Joanna Domagała-Kulawik², Rafał Krenke ${ }^{1}$, Marta Maskey-Warzęchowska ${ }^{1}$, \\ Bożena Tarchalska-Kryńska³, Paulina Krasnodębska, Ryszarda Chazan ${ }^{1}$
}

\author{
${ }^{1}$ Department of Internal Medicine, Pneumonology and Allergology, Medical University \\ of Warsaw, Poland \\ ${ }^{2}$ Department of Otolaryngology, Medical University of Warsaw, Poland \\ ${ }^{3}$ Department of Experimental and Clinical Pharmacology, Medical University \\ of Warsaw, Warsaw, Poland
}

Submitted: 2 June 2013

Accepted: 15 October 2013

Arch Med Sci 2014; 10, 5: 962-969

DOI: 10.5114 /aoms.2014.40642

Copyright (c) 2014 Termedia \& Banach

\section{Abstract}

Introduction: Efficacy of chronic cough treatment is ambiguous. The aim of the study was to analyze chronic cough alleviation after specific treatment and the relationship between cough etiology and treatment efficacy.

Material and methods: A stepwise diagnostic approach was used to diagnose cough etiology in non-smoking adults with chronic cough. In all patients specific treatment was applied. Two different questionnaires - a visual analog scale and a 5-degree scale - were used to assess cough severity before and after 4-6 months of treatment.

Results: A significant correlation between pre-treatment and post-treatment results of both questionnaires was found (Spearman coefficient 0.43, $p=0.0003$ and $0.73, p<0.0001$, respectively). Baseline questionnaire analysis revealed no differences in cough severity between patients with different cough causes or multiple cough causes. Although specific treatment resulted in a significant decrease of cough severity in the entire group, only partial improvement was noted. According to the visual analogue scale, a decrease of cough severity by at least $50 \%$ was achieved only in $54.4 \%$ of patients (37/68). Similarly, satisfactory improvement was noted in only $54.4 \%(37 / 68)$ of patients when using the 5 -point scale. There were three sub-groups of patients, in whom no relevant decrease of cough severity was observed despite treatment: patients with 1 . three coexisting cough causes, 2. non-asthmatic eosinophilic bronchitis, and 3. chronic idiopathic cough.

Conclusions: Cough severity does not depend on its etiology. Efficacy of chronic cough treatment in non-smoking patients is only moderate.

Key words: gastroesophageal reflux, upper airway cough syndrome, nonasthmatic eosinophilic bronchitis, asthma.

\section{Introduction}

Chronic cough is a common medical complaint. The most common causes of chronic (lasting over 8 weeks) cough in non-smoking adults with a normal chest radiograph include upper airway cough syndrome (UACS), gastroesophageal reflux disease (GERD), asthma and nonasthmatic eosinophilic bronchitis (NAEB). The prevalence of these conditions shows some differences [1-3].

\author{
Corresponding author: \\ Marta Dąbrowska PhD \\ Department \\ of Internal Medicine, \\ Pneumonology \\ and Allergology \\ Medical University of Warsaw \\ 1 a Banacha St \\ 02-097 Warsaw, Poland \\ Phone: +48 501157765 \\ E-mail: mdabrowska@mp.pl
}


The causative diagnosis of chronic cough is challenging. Different international guidelines presenting slightly different diagnostic approaches have been published in order to help clinicians in managing patients with chronic cough. Besides various diagnostic procedures, the assessment of the therapeutic effect of disease-specific interventions may play an important role in establishing chronic cough etiology [1]. Some authors claim that efficacy of chronic cough treatment is high and exceeds $80 \%$ [4, 5], while others state that a significant proportion of patients with chronic cough do not respond to any treatment $[6,7]$. If the diagnostic approach and empiric therapy fail to identify the cause of cough and only a modest or no therapeutic response is achieved, chronic idiopathic cough (CIC) is recognized. As hypersensitivity of the cough reflex seems to be the main cause of $\mathrm{CIC}$, the current proposed name for $\mathrm{CIC}$ is chronic cough hypersensitivity syndrome (CHS) $[6,8,9]$.

Our retrospective observations suggest that in some patients with chronic cough we were not able to achieve an improvement despite thorough diagnostic work-up and protocol-directed treatment. Experts recommend the measurement of response to cough therapy by both objective and subjective methods [2,3]. However, there are few studies estimating the degree of subjective response to chronic cough treatment. Thus, we undertook an attempt to quantify the therapeutic effects in patients with chronic cough in a prospective study.

The aim of the study was: 1) to assess the effect of protocol-directed therapy on chronic cough severity, 2) to evaluate the relationship between cough etiology and efficacy of disease-specific therapeutic interventions.

\section{Material and methods}

This prospective study was performed in patients with chronic cough, who were referred to our out-patient department between 2007 and 2009. The study protocol was approved by the Institutional Review Board of the Medical University of Warsaw. Non-smoking adult patients with cough lasting more than 8 weeks were regarded as potential candidates for the study. All patients signed an informed consent form.

\section{Diagnostic work-up}

Pre-enrollment assessment included smoking history, chest radiograph and spirometry with reversibility testing when appropriate. Active smokers, patients with significant abnormalities in the chest radiograph, as well as patients with abnormal ventilatory pattern were not included in the study group.

A set of diagnostic procedures aimed at diagnosing the etiology of chronic cough was performed in all patients meeting the primary enrollment criteria. A flow chart outlining the diagnostic work-up is shown in Figure 1.

First, data relevant to chronic cough as well as concomitant symptoms were noted in all patients. They included cough characteristics, present and past medical history, history of smoking, environmental and occupational exposure, concomitant

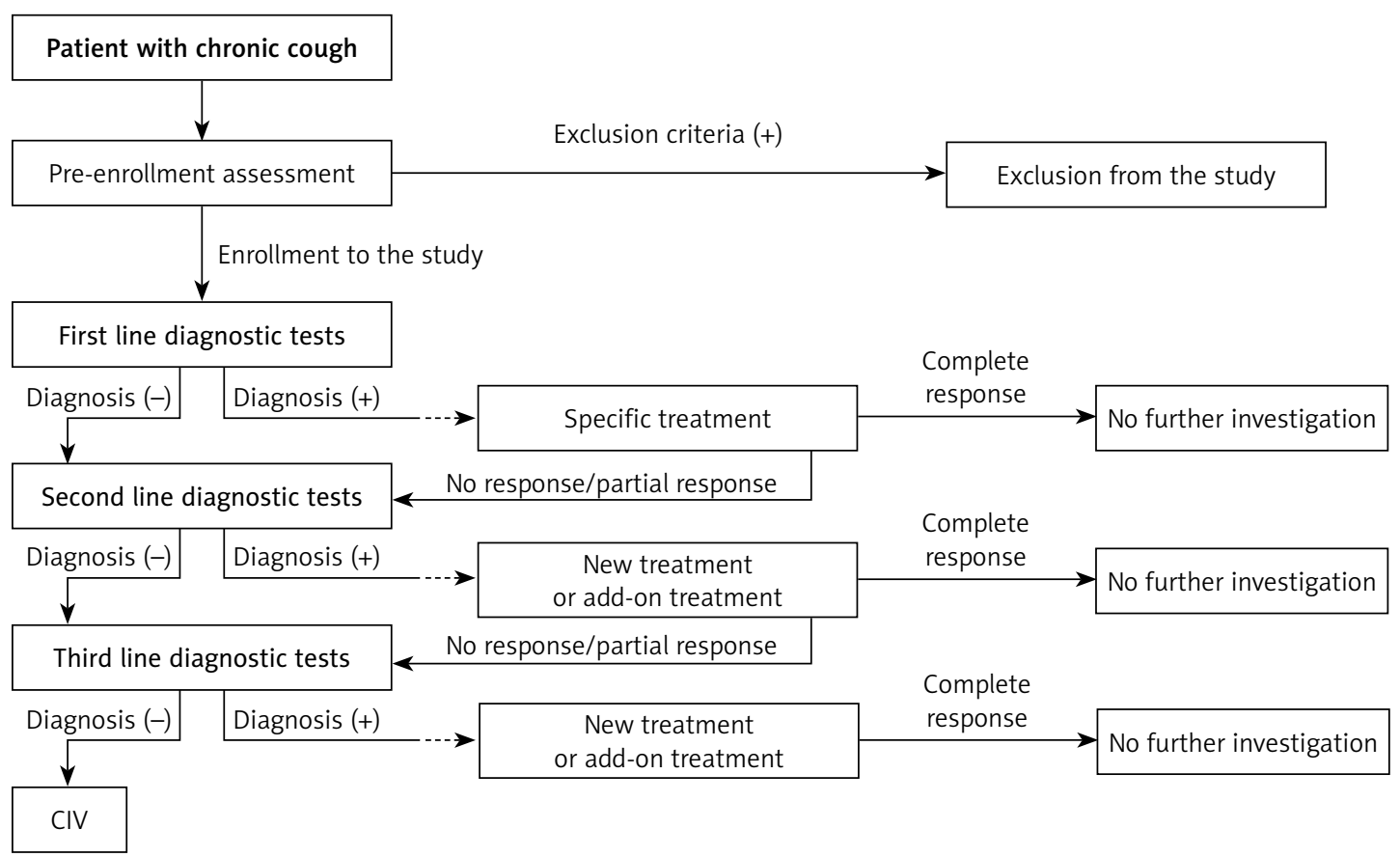

Figure 1. Flow chart outlining the diagnostic workup designed to diagnose the cause of chronic cough (see text for details) 
medication (particularly ACE inhibitors), upper respiratory tract symptoms (including post-nasal drip syndrome), symptoms suggesting GERD or asthma. All patients also underwent thorough physical examination and anterior rhinoscopy.

In all patients in whom the results of particular studies were sufficient to formulate a presumptive diagnosis of cough etiology, specific treatment was initiated, e.g. H-1 antagonists or nasal corticosteroids in UACS, diet and proton pump inhibitors in GERD, inhaled corticosteroids in asthma or NAEB. The effectiveness of such treatment was evaluated after 6-8 weeks. If no response was noted or only partial improvement was achieved, additional tests (second and third line diagnostic tests) were proposed. Second line diagnostic procedures were also performed if the cause of cough remained undetermined on the basis of the above presented studies. These included skin prick tests with common aeroallergens, methacholine inhalation challenge, induced sputum analysis, sinus CT scan, and 24-hour esophageal pH monitoring. All patients suspected of UACS were examined by an ENT specialist. These patients also underwent fiberoptic rhinoscopy, rhinomanometry, nasal cytology or videolaryngoscopy, if necessary.

Third line diagnostic procedures included high resolution computed tomography of the thorax, polysomnography, and combined multichannel intraluminal impedance-pH monitoring echocardiography.

When new potential cough causes were identified at the second and/or third level of diagnostic work-up, an adequate therapeutic intervention was added to the earlier applied treatment. The final diagnosis of chronic cough cause was based not only on the results of all diagnostic procedures, but also on the response to specific treatment.

\section{Definitions}

Diagnosis of asthma was based on the GINA guidelines [10]. Cough variant asthma (CVA) was presumed in patients with normal baseline spirometry and bronchial hyperreactivity confirmed by a $P C_{20}$ value $<4 \mathrm{mg} / \mathrm{ml}$ in the methacholine inhalation challenge [11]. The treatment of CVA included medium or high doses of inhaled corticosteroids and short acting $\beta$-agonists on demand.

Nonasthmatic eosinophilic bronchitis was diagnosed in patients with normal spirometry and negative methacholine inhalation challenge, in whom the induced sputum eosinophilia exceeded $3 \%$ of nonsquamous cells [12]. If NAEB was diagnosed, the patients were treated with medium doses of inhaled corticosteroids for at least 8 weeks.

Upper airway cough syndrome including chronic rhinitis or chronic sinusitis was based on clini- cal symptoms and confirmed by ENT specialists. Chronic sinusitis was determined on the basis of clinical signs and CT evaluation according to Lund and Mackay score [13]. Chronic rhinitis was diagnosed on the basis of clinical symptoms, and fiberoptic rhinoscopy, rhinomanometry or nasal cytology. The treatment of UACS included H-1 antagonists (promethazine) or nasal corticosteroids for at least 4 weeks.

Gastroesophageal reflux disease was assessed with 24-hour esophageal $\mathrm{pH}$ monitoring and videolaryngoscopy. Gastroesophageal reflux disease was reported if at least one of four parameters (DeMeester $\geq 14.72$, total fraction time $\mathrm{pH}<4$ [\%] $\geq 4.2$, upright $\geq 6.3$ and supine $\geq 1.2$ ) was present on the electrode placed $5 \mathrm{~cm}$ above a lower esophageal sphincter. The diagnosis of GERD-associated cough was made if cough episodes, marked by the patients on the recorder and noted down in the diary, appeared $\leq 5 \mathrm{~min}$ after the reflux $[14,15]$. Videolaryngoscopy was performed to evaluate gastropharyngeal reflux-induced changes in the laryngeal mucosa in accordance with Belafsky's reflux finding score (RFS) [16]. Some patients suspected of weakly acidic or non-acidic reflux also underwent combined multichannel intraluminal impedance-pH (MII/pH) monitoring [14]. Patients suspected or diagnosed with GERD were treated with diet and omeprazole $40 \mathrm{mg}$ b.i.d. for at least 8 weeks. Patients suspected of weakly acidic or non-acidic reflux were additionally given cisapride.

Finally, if no cause of chronic cough was found despite thorough diagnostic tests and there was no cough improvement after empiric treatment, chronic idiopathic cough was diagnosed.

\section{Cough severity measurement}

Cough severity was measured by two different, subjective questionnaires:

1. Visual analog scale;

2. 5-degree severity scale.

The visual analog scale (VAS) is a simple and frequently used method to assess the severity of difficult to measure, subjective features such as pain, dyspnea or cough on a continuous line $100 \mathrm{~mm}$ in length. The lowest extreme point (0 point) represents no cough, while the upper extreme point (100 $\mathrm{mm}$ point) corresponds to the strongest cough that the patient has ever experienced. A decrease by $50 \%$ or $20 \mathrm{~mm}$ is assumed to be relevant for cough alleviation [17].

The 5-degree severity scale is a scale evaluating cough frequency and its influence on daily activities and night rest. It is based on a cough severity scale used by Park et al. [17]. The lowest score (score 0) indicates no cough, while the highest score (score 4) corresponds to severe cough with an impact on daily everyday activity and 
causing sleep disturbance. A result lower by at least 1 point was considered significant for symptom improvement.

Both questionnaires were performed twice: during the first visit (before any treatment was introduced) and after 4-6 months of treatment.

\section{Statistical analysis}

Data are shown as median and range. The relative contribution of different cough causes was shown in two ways: as a percentage of patients with a particular cough cause and as a percentage of all diagnosed causes of cough. Correlation between the results of two questionnaires was analyzed by Spearman's coefficient. Dependence statistics was applied to estimate the relationship between cough severity and its causes or number of cough causes. For the 5-point scale, Fisher's exact test was used. The Kruskal-Wallis test was used for VAS to assess the relationship between cough severity and its etiology and to analyze the influence of number of cough causes on cough severity.

\section{Results}

Seventy-five patients were enrolled in the study; 68 (90.6\%) returned pre- and post-treatment questionnaires and were included in the final analysis. Forty patients (58.8\%) had already been diagnosed or unsuccessfully treated prior to the first visit at our institution. Baseline characteristics of the study group are presented in Table I. A single cough etiology was diagnosed in 19 patients (28\%), two cough causes were diagnosed in 37 patients (54.5\%), while in 12 patients (17.6\%) three coexisting cough causes were diagnosed (Table II).

Gastroesophageal reflux disease was the most common cause of chronic cough and was diagnosed in 48 patients (48/68, 71\%) (Table II).

Table II. Etiology of chronic cough

\begin{tabular}{|c|c|c|c|c|}
\hline Cough cause & $\begin{array}{c}\text { Single cough } \\
\text { etiology }(n=19)\end{array}$ & $\begin{array}{l}\text { Multiple cough } \\
\text { etiology }(n=49)\end{array}$ & $\begin{array}{l}\text { Total number (percentage } \\
\text { and } 95 \% \mathrm{Cl} \text { ) of patients with } \\
\text { respective cough etiology }\end{array}$ & $\begin{array}{l}\text { Percentage of all } \\
\text { diagnosed causes } \\
\text { of cough (129) }\end{array}$ \\
\hline GERD & 8 & 40 & $\begin{array}{c}48(71 \%) \\
(0.58-0.81)\end{array}$ & $37 \%$ \\
\hline UACS & 5 & 31 & $\begin{array}{c}36(53 \%) \\
(0.40-0.65)\end{array}$ & $28 \%$ \\
\hline CVA & 1 & 17 & $\begin{array}{c}18(26 \%) \\
(0.16-0.38)\end{array}$ & $14 \%$ \\
\hline NAEB & 1 & 8 & $\begin{array}{c}9(13 \%) \\
(0.06-0.23)\end{array}$ & $7 \%$ \\
\hline Other causes & 0 & 14 & $\begin{array}{c}14(21 \%) \\
(0.14-0.31)\end{array}$ & $11 \%$ \\
\hline $\mathrm{CIC}$ & 4 & 0 & $\begin{array}{c}4(6 \%) \\
(0.01-0.12)\end{array}$ & $3 \%$ \\
\hline
\end{tabular}

GERD - gastroesophageal reflux disease, UACS - upper airway cough syndrome, CVA - cough variant asthma, NAEB - nonasthmatic eosinophilic bronchitis, CIC - chronic idiopathic cough
Gastroesophageal reflux disease was diagnosed ic treatment $(19 / 48,39.5 \%)$, results of 24 -hour esophageal $\mathrm{pH}$ monitoring $(19 / 48,39.5 \%)$ or impedance monitoring (10/48, 21\%). Median Belafsky's RFS in videolaryngoscopy in this group was 11 (range: 6-15).

Upper airway cough syndrome was diagnosed in 36 patients (53\%) and was the second most common cause of chronic cough in the sub-group of patients with a single cough cause $(26 \%)$. There was a significant predominance of chronic rhinitis over chronic rhinosinusitis in patients with UACS (30 patients vs. 6 patients), respectively. Detailed data on UACS etiology are presented in Table III.

Cough variant asthma and NAEB were diagnosed in 18 and 9 patients, respectively. Other causes of cough were diagnosed in 14 patients (21\%): side effects of ACE inhibitors $(n=4)$, bronchiectasis $(n=2)$, obstructive sleep apnea syndrome $(n=2)$, heart failure $(n=2)$, pulmonary embolism $(n=1)$, airway colonization with Mycobacterium xenopi (no radiological sign of pulmonary involvement) $(n=2)$ and arrhythmia-induced cough $(n=1)$. Chronic idiopathic cough was diagnosed in 4 (6\%) patients. More data on the distribution of specific cough etiologies are presented in Table II.

Table I. Baseline characteristics of study group $(n=68)$

\begin{tabular}{|lc|}
\hline Parameter & Result \\
\hline Age, median (range) [years] & $49.6(21-81)$ \\
\hline Gender - female, $n(\%)$ & $49(72)$ \\
\hline BMI, median (range) $\left[\mathrm{kg} / \mathrm{m}^{2}\right]$ & $28.4(19.0-33.4)$ \\
\hline $\begin{array}{l}\text { Duration of cough, median } \\
\text { (range) }\end{array}$ & $\begin{array}{c}24 \text { months } \\
\text { (10 weeks }-30 \text { years) }\end{array}$ \\
\hline \begin{tabular}{l} 
Never-smokers/ex-smokers \\
\hline
\end{tabular} & $56 / 12$ \\
\hline
\end{tabular}
based on either subjective response to empir- 
M. Dąbrowska, E.M. Grabczak, M. Arcimowicz, A. Domeracka-Kołodziej, J. Domagała-Kulawik, R. Krenke, M. Maskey-Warzęchowska, B. Tarchalska-Kryńska, P. Krasnodębska, R. Chazan

Table III. Etiology of upper airway cough syndrome

\begin{tabular}{|lc|}
\hline Cause of UACS & $\begin{array}{c}\text { Number of patients } \\
\text { (percentage of all } \\
\text { patients with UACS) }\end{array}$ \\
\hline Perennial allergic rhinitis & $\begin{array}{c}10(28 \%) \\
\text { Dust mites - } 8 \\
\text { Other allergens - } 2\end{array}$ \\
\hline $\begin{array}{l}\text { Nonallergic rhinitis with } \\
\text { eosinophilia }\end{array}$ & $4(11 \%)$ \\
\hline Nonallergic rhinopathy & $5(14 \%)$ \\
\hline Atrophic rhinitis & $3(8 \%)$ \\
\hline Drug-induced rhinitis & $2(0.5 \%)$ \\
\hline Rhinosinusitis & $6(17 \%)$ \\
\hline Unknown & $6(17 \%)$ \\
\hline UACS - upper airway cough syndrome & \\
\hline
\end{tabular}

A significant correlation between pre-treatment results of both questionnaires was found (Spearman coefficient $0.43, p=0.0003$ ). Similarly, post-treatment analysis revealed a highly significant correlation between the scores measured by two different questionnaires (Spearman coefficient $0.73, p<0.0001$ ).
Baseline questionnaire analysis revealed no differences in cough severity between patients with different cough causes or multiple cough causes.

Treatment resulted in a significant decrease in cough severity in the entire group. The median differences in the VAS and 5-point scale were 20.0 millimeters and 1.0 point respectively $(p<0.001)$. However, only partial improvement was noted. According to the VAS, a decrease of cough severity of $50 \%$ or more was achieved only in 37 patients $(37 / 68,54.4 \%)$. Only in 6 patients $(9 \%)$ was the post-treatment VAS score lower than $10 \mathrm{~mm}$. Similarly, according to the 5-point scale, a satisfactory improvement was found only in 37 (54.5\%) patients (Table IV). There were three groups of patients in whom no relevant decrease of cough severity was observed despite treatment: 1) patients with three coexisting cough causes, 2) patients with NAEB, and 3) patients with chronic idiopathic cough (Table IV).

\section{Discussion}

Although the main causes of chronic cough in our patients were similar to those reported by other authors [1, 18], the proportion of GERD $(71 \%)$

Table IV. Difference in cough severity after treatment measured by VAS and 5-point scale according to cough etiology

\begin{tabular}{|c|c|c|c|c|c|}
\hline \multicolumn{2}{|c|}{ Number of patients } & \multirow{2}{*}{$\begin{array}{c}\text { 5-point scale } \\
1 \\
\end{array}$} & \multirow{2}{*}{$\begin{array}{l}\text { VAS } \\
20\end{array}$} & \multirow{4}{*}{$\begin{array}{c}\text { Number of patients } \\
\text { with reduction } \\
\text { in VAS > 50\% } \\
37 / 68 \\
54.5 \%\end{array}$} & \multirow{4}{*}{$\begin{array}{c}\text { Number of patients } \\
\text { with reduction } \\
\text { in VAS } \geq 20 \mathrm{~mm} \\
37 / 68 \\
54.5 \%\end{array}$} \\
\hline All & Median & & & & \\
\hline & $95 \% \mathrm{Cl}$ & {$[-1]$ to 3.0} & {$[-23.5]$ to 71} & & \\
\hline & $p$ & $<0.001$ & $<0.001$ & & \\
\hline \multirow{3}{*}{$\begin{array}{l}\text { GERD } \\
n=48\end{array}$} & Median & 1 & 20 & \multirow{3}{*}{$\begin{array}{c}27 / 48 \\
56 \%\end{array}$} & \multirow{3}{*}{$\begin{array}{c}27 / 48 \\
56 \%\end{array}$} \\
\hline & $95 \% \mathrm{Cl}$ & {$[-1]$ to 3} & {$[-23]$ to 66} & & \\
\hline & $p$ & 0.009 & 0.0024 & & \\
\hline \multirow{3}{*}{$\begin{array}{l}\text { UACS } \\
n=36\end{array}$} & Median & 1 & 20 & \multirow{3}{*}{$\begin{array}{c}17 / 36 \\
47 \%\end{array}$} & \multirow{3}{*}{$\begin{array}{c}19 / 36 \\
53 \%\end{array}$} \\
\hline & $95 \% \mathrm{Cl}$ & {$[-1]$ to 3} & {$[-23]$ to 57} & & \\
\hline & $p$ & 0.014 & 0.0014 & & \\
\hline \multirow{3}{*}{$\begin{array}{l}\text { CVA } \\
n=18\end{array}$} & Median & 1 & 30 & \multirow{3}{*}{$\begin{array}{c}10 / 18 \\
55 \%\end{array}$} & \multirow{3}{*}{$\begin{array}{c}12 / 18 \\
66 \%\end{array}$} \\
\hline & $95 \% \mathrm{Cl}$ & 0 to 3 & {$[-20]$ to 75} & & \\
\hline & $p$ & 0.031 & 0.023 & & \\
\hline \multirow{3}{*}{$\begin{array}{l}\text { NAEB } \\
n=9\end{array}$} & Median & 1 & 19 & \multirow{3}{*}{$\begin{array}{c}4 / 9 \\
44 \%\end{array}$} & \multirow{3}{*}{$\begin{array}{c}4 / 9 \\
44 \%\end{array}$} \\
\hline & $95 \% \mathrm{Cl}$ & 0 to 3 & 4 to 43 & & \\
\hline & $p$ & 0.25 & 0.062 & & \\
\hline \multirow{3}{*}{$\begin{array}{l}\text { Other } \\
n=14\end{array}$} & Median & 1 & 25 & \multirow{3}{*}{$\begin{array}{l}8 / 14 \\
57 \%\end{array}$} & \multirow{3}{*}{$\begin{array}{l}8 / 14 \\
57 \%\end{array}$} \\
\hline & $95 \% \mathrm{Cl}$ & 0 to 3 & [-5] to 71 & & \\
\hline & $p$ & 0.007 & 0.004 & & \\
\hline \multirow{3}{*}{$\begin{array}{l}\mathrm{CIC} \\
n=4\end{array}$} & Median & 0 & 0 & \multirow{3}{*}{$\begin{array}{c}1 / 4 \\
25 \%\end{array}$} & \multirow{3}{*}{$\begin{array}{c}1 / 4 \\
25 \%\end{array}$} \\
\hline & $95 \% \mathrm{Cl}$ & {$[-1]$ to 0} & {$[-21]$ to 20} & & \\
\hline & $p$ & 0.93 & 0.85 & & \\
\hline
\end{tabular}

GERD - gastroesophageal reflux disease, UACS - upper airway cough syndrome, CVA - cough variant asthma, NAEB - nonasthmatic eosinophilic bronchitis, CIC - chronic idiopathic cough 
- the most common cause of cough in our study - was higher than that found in earlier publications. The frequent diagnosis of GERD could have been related to our diagnostic protocol, which involved several different diagnostic methods directed at recognizing GERD as a cause of chronic cough. In our previous study, we also found a high prevalence of GERD in patients with chronic cough and this was irrespective of the diagnostic protocol [19]. Although the prevalence of GERD in the majority of other studies was lower (20-40\%), several authors also reported GERD as the most frequent cause of chronic cough [20-22].

The second most common cause of chronic cough in our study was UACS. A complex otorhinolaryngological approach enabled the diagnosis of different types of the disease (Table III). The prevalence of rhinosinusitis was relatively low, and this corresponds with the observation of Watalet et al., who found that chronic cough is definitely more frequent in patients with rhinitis than rhinosinusitis [23].

Although patients with clinical symptoms consistent with asthma and reversible airflow limitation were excluded at the initial phase of enrollment, the percentage of asthmatics in our study group was $26 \%$. This result is fully consistent with the ACCP statement that asthma is one of the three most common cough causes with prevalence between $24 \%$ and $29 \%$ in adult non-smokers with chronic cough [24]. Due to the selection criteria, only cough variant asthma was diagnosed in patients participating in our study. We might suppose that if we had not applied clinical and spirometric criteria excluding a majority of patients with asthma, the percentage of asthmatics in our study group would have been even higher.

With regard to the primary goal of our study, we found that in the entire group of patients, an appropriate causal treatment resulted in a significant decrease of cough severity. However, it should be emphasized that only a partial response was observed. According to the VAS and the 5-point scale questionnaire, an important decrease in cough severity was achieved in approximately $55 \%$ of patients. Interestingly, very high agreement between the results of two different questionnaires (pre-treatment, as well as post-treatment) was noted.

The reason for only moderate improvement after a cause-directed therapeutic intervention is an interesting point for discussion. One potential explanation for this phenomenon would be a misdiagnosis of the true cough causes. At the early phase of management, it is difficult to prove a causative relationship between chronic cough and various clinical conditions. According to Yu et al., a discrepancy between the presumptive and final diagnosis of the cause of chronic cough may be common and may lead to overtreatment [25]. Thus, we cannot exclude that our presumptive diagnosis of cough underlying disease was wrong, resulting in comorbidity-directed intervention rather than true causative cough treatment. On the other hand, the efficacy of chronic cough treatment has also been questioned by other authors $[6,26]$. Our results are similar to those reported by Levine. This author documented a complete response to treatment in $41 \%$, partial response in $32 \%$ and no response in $16 \%$ of patients with chronic cough [26]. Recent papers suggest that the main factor in the pathomechanism of chronic cough is hypersensitivity of the cough reflex, while GERD, UACS or asthma may only be triggers and not the real causes of cough. Therefore, if standard treatment of chronic cough is unsuccessful, the therapy of hypersensitive cough reflex should be added [27]. To make things even more difficult, there is neither a widely accepted standard method of measurement of cough treatment efficacy nor an unequivocal threshold of significant improvement of cough as far as subjective scales are concerned. Besides, one recent paper suggests that the results of subjective cough questionnaires do not correspond to objective methods such as cough monitoring techniques [28]. It seems, therefore, that the importance of subjective methods of measuring cough severity, an issue crucial for the patient, is comparable to that of objective methods [29]. Thus, we tried to estimate the subjective response to cough therapy in our patients. According to our knowledge, this is the first study which evaluated any potential relationships between the degree of response to therapy and cough causes.

Although there are a lot of studies on the efficacy of GERD treatment, their results are ambiguous. A meta-analysis of treatment efficacy with proton-pump inhibitors in patients with cough due to GERD failed to confirm their effectiveness [30]. Disorders of esophageal motility are suspected to be an important factor in pathogenesis of GERD-related cough. On the other hand, results of esophageal multichannel impedance monitoring did not reveal a difference in the frequency of acid and non-acid reflux between chronic cough patients and healthy subjects [31, 32].

As UACS is heterogeneous in nature, the treatment of this entity is difficult. The most common causes of UACS are perennial allergic rhinitis, nonallergic rhinitis with eosinophilia, nonallergic rhinopathy, atrophic or senile rhinitis and drug-induced or hormone-induced rhinitis or rhinosinusitis. Except for allergic rhinitis, the diagnosis and treatment of all the above is difficult and only partially successful $[33,34]$. Besides, a decrease of 
cough severity after antihistaminic drugs in patients with UACS does not prove that rhinitis is the cause of cough, because antihistaminic drugs may influence the cough reflex, not only rhinitis itself [32].

According to guidelines on cough management, NAEB is responsible for chronic cough in about $10-20 \%$ of adult patients [12]. Nonasthmatic eosinophilic bronchitis diagnosis requires successful sputum induction, but this may be difficult to obtain as many patients are unable to produce adequate sputum samples [35]. In our study, NAEB was diagnosed in $13 \%$ of subjects. It is striking that the NAEB group was one of the three groups in which we did not achieve a significant decrease of cough severity after 4-6 months of treatment. Importantly, lack of improvement was noted when comparing pre- and post-treatment scores in both questionnaires. Such results are in opposition to the common opinion that NAEB responds well to treatment with inhaled steroids [12, 17]. Lack of success may result from moderate doses of inhaled corticosteroids used in our patients (the average daily dose of budesonide was $400 \mu \mathrm{g}$ ). The lack of patients' compliance may also be responsible for unsuccessful treatment in this group. It should be emphasized, however, that limited treatment efficacy of NAEB (or even asthma) has also been reported by other authors [36, 37]. In an observational study by Berry et al., cough and induced sputum eosinophilia persisted in some patients with NAEB despite treatment with inhaled corticosteroids. Moreover, $25 \%$ of these patients eventually developed airway obstruction (COPD in $16 \%$ and asthma in 9\%) [36].

The group of patients with three coexisting cough causes was the second study group in which no relevant cough alleviation, despite complex treatment, was noted. One may intuitively suppose that the more complex the cough etiology is, the worse is the effect of cough treatment. However, according to results by Levine, the efficacy of chronic cough treatment did not depend on the number of cough causes [26].

The third group in which no relevant therapeutic effect was observed consisted of subjects with $\mathrm{CIC}$. This was to be expected, as the cause of cough in these patients was not determined. The importance of $\mathrm{CIC}$ has been emphasized in some recent papers. According to the definition, $\mathrm{CIC}$ is recognized if no specific diagnosis is reached despite thorough investigation and if trials of specific therapy do not decrease cough intensity $[8,32]$. The prevalence of $\mathrm{CIC}$ differs significantly among studies and may be as high as $42 \%$ [6]. In our study group, CIC (defined as no diagnosed cough cause) was diagnosed in 4 patients (6\%). Cough reflex hypersensitivity is usually considered as the major cough mechanism in these patients $[9,31]$.
It may be interesting that in some patients with $\mathrm{CIC}$, coexistence of autoimmunological disorders has been documented [38]. Further studies on the pathogenesis of $\mathrm{CIC}$ are certainly needed. There is an ongoing search for new medications and methods of CIC management. One of the treatment options is speech language training, as it reduces the number of cough episodes and decreases cough reflex hypersensitivity [39].

We are aware of several limitations of our study. First, we did not use objective methods of cough severity measurements such as cough monitors and capsaicin or citric acid inhalation cough challenge $[29,31,40]$. We did not apply these methods simply because they were not available in our institution at the time of study onset and we had no experience with their use. However, there is evidence that subjective and objective methods of cough severity measurements are complementary $[28,32]$. Second, the cough etiology was determined mainly on examination results. That could have led to overdiagnosis of cough etiology by recognition of comorbidities that coexist with cough, but were not its actual cause. Third, the limit of significant decrease in cough severity according to the VAS after treatment set at 50\% was arbitrary. Finally, since the study was performed in a tertiary referral center and included patients who had troublesome cough despite previous interventions, one may suspect selection bias influencing the results of treatment.

In conclusion, chronic cough severity does not depend on its etiology. Despite detailed thorough procedures and 4-6 months of treatment, efficacy of chronic cough treatment in our patients was only moderate. The decrease of cough severity was more than $50 \%$ in only about $55 \%$ of patients.

\section{References}

1. Irwin RS, Baumann MH, Bolser DC, et al. Diagnosis and management of cough. ACCP evidenced-based clinical practice guidelines. Chest 2006; 129 (suppl): 1s-23s.

2. Morice $\mathrm{AH}$ and Committee Members. The diagnosis and management of chronic cough. Eur Respir J 2004; 24 : 481-92.

3. Morice AH, LMcGarvey, Pavord I, et al. Recommendations for the management of cough in adults. Thorax 2006; 61(suppl 1): i1-i24.

4. McGarvey LPA, Heaney LG, Lawson JT, et al. Evaluation and outcome of patients with chronic non-productive cough using a comprehensive diagnostic protocol. Tho$\operatorname{rax} 1998 ;$ 53: 738-43.

5. Mello CJ, Irwin RS, Curley FJ. Predictive values of the character, timing and complications of chronic cough in diagnosing its cause. Arch Intern Med 1996; 156: 997-1003.

6. Haque RA, Usmain OS, Barnes PJ. Chronic idiopathic cough. A discrete clinical entity? Chest 2005; 127: 1710-3.

7. Kastelik JA, Aziz I, Ojoo JC, et al. Investigation and management of chronic cough using a probability-based algorithm. Eur Respir J 2005; 25: 235-43. 
8. Birring SS. New concepts in the management of chronic cough. Pulm Pharmacol Ther 2011; 24: 334-8.

9. Morice AH. The cough hypersensitivity syndrome: a novel paradigm for understanding cough. Lung 2010; 188 (suppl 1): S87-90.

10. Global strategy for asthma management and prevention (update 2012): Global Initiative for Asthma (GINA). URL: http://www.ginasthma.org

11. Lougheed MD, Turcotte SE, Fisher T. Cough variant asthma: lessons learned from deep inspirations. Lung 2012; 190: 17-22.

12. Brightling CE. Chronic cough due to nonasthmatic eosinophilic bronchitis. ACCP evidence-based clinical practice guidelines. Chest 2006; 129: 116S-21S.

13. Lund VJ, Mackay IS. Staging in rhinosinusitis. Rhinology 1993; 31: 183-4.

14. Tutuian R. Reflux monitoring: current status. Curr Gastreneterol Rep 2008; 10: 263-70.

15. Irwin RS. Chronic cough due to gastroesophageal reflux disease: ACCP evidence based clinical practice guidelines. Chest 2006; 129: 82S-94S.

16. Belafsky PC, Postman GN, Koufman JA. The validity and reliability of the reflux finding score (RFS). Laryngoscope 2001; 111: 1313-7.

17. Park SW, Lee YM, Jang AS, et al. Development of chronic airway obstruction in patients with eosinophilic bronchitis. Chest 2004; 125: 1998-2004.

18. Chung KF, Pavord ID. Prevalence, pathogenesis and causes of chronic cough. Lancet 2008; 371: 1364-74.

19. Grabczak EM, Dąbrowska M, Krenke R, et al. Does the established cause of chronic cough depend on diagnostic approach? J Physiol Pharmacol 2008; 59: 285-96.

20. Palombini BC, Villanova CAC, Araujo E, et al. A pathogenic triad in chronic cough: asthma, postnasal drip syndrome, and gastroesophageal reflux disease. Chest 1999; 116: 279-84.

21. French CL, Irwin RS, Curlej FJ, Krikorian CJ. Impact of chronic cough on quality of life. Arch Intern Med 1998; 158: 1657-61.

22. Carney IK, Gibson PG, Murree-Allen K, et al. A systematic evaluation of mechanism in chronic cough. Am J Respir Crit Care Med 1997; 156: 211-6.

23. Watalet JB, Van Ziele T, Brusselle G. Chronic cough in upper airway diseases. Respir Med 2010; 104: 652-7.

24. Dicpinigaitis PV. Chronic cough due to asthma. ACCP evidence-based clinical practice guidelines. Chest 2006; 129: 75S-9S.

25. Yu Li, Qiu Z, Wei W, et al. Discrepancy between presumptive and definite causes of chronic cough. Chin Med J 2011; 124: 4138-43.

26. Levine BM. Systemic evaluation and treatment of chronic cough in a community setting. Allergy Asthma Proc 2008; 29: 336-42.

27. Ryan N, Birring S, Gibson P. Gabapentin for refractory chronic cough: a randomized, double-blind, placebo-controlled trial. Lancet 2012; 380: 1583-9.

28. Kelsall A, Houghton LA, Jones $\mathrm{H}$, et al. A novel approach to studying the relationship between subjective and objective measures of cough. Chest 2011; 139: 569-75.

29. Leconte S, Ferrant D, Dory V, et al. Validated methods of cough assessment: a systematic review of the literature. Respiration 2011; 81: 161-73.

30. Chang AB, Lasserson TJ, Kilijander TO, et al. Systemic review and meta- analysis of randomized controlled trials of gastro-esophageal reflux interventions for chronic cough associated with gastro-esophageal reflux. BMJ 2006; 332: 11-7.
31. Decalmer SC, Webster D, Kelsall AA, et al. Chronic cough: how do cough reflex sensitivity and subjective assessments correlate with objective cough counts during ambulatory monitoring? Thorax 2007; 62: 329-34.

32. Birring SS. Controversies in the evaluation and management of chronic cough. Am J Respir Crit Care Med 2011; 183: 708-15.

33. Pratter MR. Chronic upper airway cough syndrome secondary to rhinosinus diseases. ACCP evidence-based clinical practice guidelines. Chest 2006; 129: 63S-71S.

34. Tran NP, Vickery J, Blaiss MS. Management of rhinitis: allergic an non-allergic. Allergy Asthma Immunol Res 2011; 3: 148-56.

35. Majewski S, Cichocki P, Stępnicka-Bindemann M, Górski P. Asthma control, quality of life and successful sputum induction. Arch Med Sci 2011; 7: 840-3.

36. Berry MA, Hargadon B, McKenna S, et al. Observational study of the natural history of eosinophilic bronchitis. Clin Exp Allergy 2005; 35: 598-601.

37. Marandi Y, Farahi N, Hashjin GS. Asthma: beyond corticosteroid treatment. Arch Med Sci 2013; 9: 521-6.

38. Birring SS, Brightling CE, Symon FA, et al. Idiopathic chronic cough: association with organ specific autoimmune disease and bronchoalveolar lymphocytosis. Thorax 2003; 58: 1066-70.

39. Ryan NM, Vertigan AE, Bone S, et al. Cough reflex sensitivity improves with speech language pathology management of refractory chronic cough. Cough 2010; 6: 5.

40. Morice AH, Fontana GA, Belvisi MG, et al. ERS guidelines on the assessment of cough. Eur Respir J 2007; 29: $1256-76$. 